\title{
Semi-blind channel estimation using knowledge of a pulse shape for faster- than-Nyquist signaling
}

\author{
Yusuke Setoguchi, Teruyuki Miyajima ${ }^{a}$, and Yoshiki Sugitani \\ Graduate School of Science and Engineering, Ibaraki University, \\ 4-12-1 Nakanarusawa, Hitachi-shi, Ibaraki 316-8511, Japan \\ a)teruyuki.miyajima.spc@vc.ibaraki.ac.jp
}

\begin{abstract}
In this paper, we propose a semi-blind channel estimation method for faster-than-Nyquist (FTN) signaling. The proposed method combines the least-squares (LS) method using training symbols with a blind estimation method using knowledge of the pulse shape. It is shown that the proposed semi-blind method requires fewer training symbols than the LS method, and it is superior to the blind method regardless of the packing ratio of FTN signaling.
\end{abstract}

Keywords: faster-than-Nyquist signaling, channel estimation, semi-blind estimation

Classification: Wireless Communication Technologies

\section{References}

[1] A. Prlja, J. B. Anderson, and F. Rusek, "Receivers for faster-than-Nyquist signaling with and without turbo equalization," Proc. IEEE Int. Symp. Inf. Theory, Toronto, Canada, pp. 464-468, Jul. 2008. DOI:10.1109/ISIT.2008. 4595029

[2] H. Fukumoto and K. Hayashi, "Overlap frequency domain equalization for faster-than-Nyquist signaling," http://arxiv.org/abs/1509.00562, accessed Jan. 1, 2019.

[3] S. Sugiura, "Frequency-domain equalization of faster-than-Nyquist signaling," IEEE Wireless Commun. Lett., vol. 2, no. 5, pp. 555-558, 2013. DOI:10.1109/ WCL.2013.072313.130408

[4] T. Ishihara and S. Sugiura, "Iterative frequency-domain joint channel estimation and data detection of faster-than-Nyquist signaling," IEEE Trans. Wireless Commun., vol. 16, no. 9, pp. 6221-6231, Sep. 2017. DOI:10.1109/TWC.2017. 2721367

[5] N. Wu, W. Yuan, G. Guo, and J. Kuang, "A hybrid BP-EP-VMP approach to joint channel estimation and decoding for FTN signaling over frequency selective fading channels," IEEE Access, vol. 5, pp. 6849-6858, May 2017. DOI:10.1109/ACCESS.2017.2702571

[6] Z. Ding, "Multipath channel identification based on partial system information," IEEE Trans. Signal Process., vol. 45, no. 1, pp. 235-240, Jan. 1997. DOI:10. $1109 / 78.552222$ 


\section{Introduction}

Faster-than-Nyquist (FTN) signaling is a non-orthogonal transmission scheme that sends pulses at a rate greater than the Nyquist rate, and it can increase the data rate without expanding bandwidth. However, due to the non-orthogonal nature, FTN signaling essentially suffers from inter-symbol interference (ISI). In frequencyselective channel scenarios, the effect of ISI is significant, so a powerful and robust equalizer is necessary. To date, several equalization methods for FTN signaling have been proposed $[1,2,3]$. Because the performance of these equalization methods depends on the accuracy of the estimated channel, channel estimation is a critical task for FTN signaling.

It is inefficient to estimate the total channel composed of a frequency-selective physical channel and a Nyquist pulse. Because the receiver knows the pulse shape used, it is sufficient to estimate only the physical channel $[4,5]$. In [4], a frequencydomain channel estimation method for cyclic-prefixed (CP) FTN signaling was proposed. Training symbols embedded in the $\mathrm{CP}$ were used to estimate the channel based on the minimum mean square error criterion. Its disadvantage is the data rate loss caused by the insertion of CP. In [5], a time-domain channel estimation method using a message-passing algorithm was proposed. In this method, joint channel estimation and data decoding were performed in an iterative manner. To simplify iterative processing, a channel estimate initially obtained by the least-squares (LS) method using the training symbols was refined. Its disadvantage is the computational complexity increase due to the joint estimation/detection processing and the iterative processing.

For Nyquist signaling, there have been various blind channel estimation methods that require no training symbols; however, most of them attempt to estimate the total channel. In [6], a blind estimation method that estimated only the physical channel by using knowledge of the pulse shape was proposed, and its superiority to the corresponding method that ignores the known pulse shape was reported. Although this approach can be applied to FTN signaling in principle, such studies have not yet been done.

In this paper, we propose a semi-blind channel estimation method for FTN signaling, which is a combination of the LS estimation method using training symbols and the blind estimation method in [6]. The proposed method can improve the performance compared to the blind method with the help of training symbols, and it can reduce the number of training symbols compared to the LS method.

\section{System model}

Fig. 1 shows the system model of FTN signaling. At the transmitter, data symbol $s[k]$ is transmitted after passing through a transmit filter $g_{\mathrm{f}}(t)$ every $T=\gamma T_{0}$ seconds, where $0<\gamma \leq 1$ is the packing ratio and $T_{0}$ is the symbol period of Nyquist signaling. The transmitted signal goes through an unknown frequencyselective physical channel $c(t)$, which has finite support $\left[0, L T_{0}\right)$. At the receiver, the received signal passed through a receive filter $g_{\mathrm{f}}(t)$ is given by

$$
r(t)=\sum_{k} s[k] h(t-k T)+\eta(t)
$$




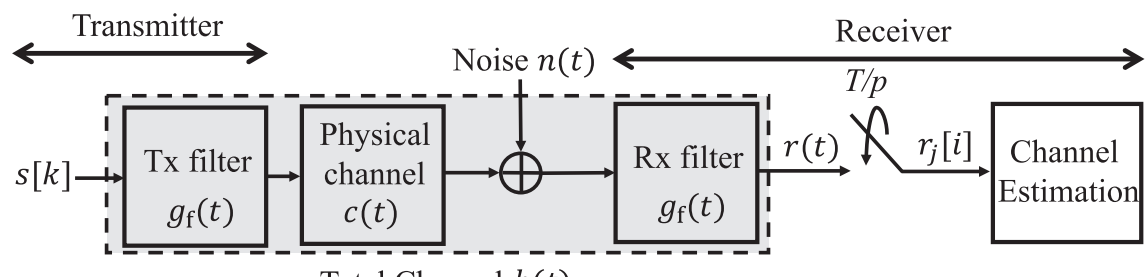

Total Channel $h(t)$

Fig. 1. System model.

where $h(t)=\int c(\tau) g(t-\tau) d \tau$ is the "total" channel impulse response (IR) that includes the physical channel $c(t)$ and the combined transmit/receive filter $g(t)=\int g_{\mathrm{f}}(\tau) g_{\mathrm{f}}(t-\tau) d \tau$, which is a Nyquist pulse truncated to $\left[-N T_{0}, N T_{0}\right]$. In this case, $\eta(t)=\int n(\tau) g_{\mathrm{f}}(t-\tau) d \tau$ is a colored noise process with the autocorrelation function $E\left[\eta(t) \eta^{*}(t+\tau)\right]=N_{0} g(\tau)$, where $n(t)$ is a complex-valued white Gaussian random process of PSD $\frac{N_{0}}{2}$.

The received signal is oversampled with an interval of $\frac{T}{p}$, where $p$ is an integer oversampling factor. By defining $r_{j}[i]=r\left(\left(i+\frac{j}{p}\right) T\right), h_{j}[i]=h\left(\left(i+\frac{j}{p}\right) T\right)$ and $\eta_{j}[i]=\eta\left(\left(i+\frac{j}{p}\right) T\right)$, we obtain the $(i p+j)$ th received sample as

$$
r_{j}[i]=\sum_{l=-\tilde{N}}^{\tilde{N}+\tilde{L}} h_{j}[l] s[i-l]+\eta_{j}[i], \quad j=0, \cdots, p-1
$$

where $\tilde{N}=\left\lceil\frac{N}{\gamma}\right\rceil$ if $\left\lceil\frac{N}{\gamma}\right\rceil-\frac{N}{\gamma}<\frac{p-1}{p} ;$ otherwise, $\tilde{N}=\left\lfloor\frac{N}{\gamma}\right\rfloor$, and $\tilde{L}=\left\lceil\frac{L-1}{\gamma}\right\rceil$ if $\left\lceil\frac{L-1}{\gamma}\right\rceil-\frac{L-1}{\gamma}<\frac{p-1}{\gamma p}$; otherwise, $\tilde{L}=\left\lfloor\frac{L-1}{\gamma}\right\rfloor$. We define the total channel vector as $\mathbf{h}=\left[\mathbf{h}^{T}[-\tilde{N}] \cdots \mathbf{h}^{T}\left[L_{h}-\tilde{N}-1\right]\right]^{T} \in \mathbb{C}^{L_{h} p \times 1}$, where $\mathbf{h}[l]=\left[h_{0}[l] \cdots h_{p-1}[l]\right]^{T}$ and $L_{h}=2 \tilde{N}+\tilde{L}+1$. We can write the $i$ th received sample vector $\mathbf{r}[i]=$ $\left[r_{0}[i] \cdots r_{p-1}[i]\right]^{T}$ as

$$
\mathbf{r}[i]=\mathbf{S}[i] \mathbf{h}+\boldsymbol{\eta}[i]
$$

where $\mathbf{S}[i]=[\overline{\mathbf{S}}[i+\tilde{N}] \cdots \overline{\mathbf{S}}[i-\tilde{N}-\tilde{L}]] \in \mathbb{C}^{p \times L_{h} p}$ is the transmit symbol matrix consisting of the diagonal matrices $\overline{\mathbf{S}}[d] \in \mathbb{C}^{p \times p}$ whose diagonal elements are $s[d]$ and $\boldsymbol{\eta}[i]=\left[\eta_{0}[i] \cdots \eta_{p-1}[i]\right]^{T}$. Stacking $\mathbf{r}[i]$ over $M$ symbols, we have

$$
\mathbf{r}_{M}=\left[\mathbf{r}^{T}[i] \cdots \mathbf{r}^{T}[i-M+1]\right]^{T}=\mathbf{S}_{M} \mathbf{h}+\boldsymbol{\eta}_{M} \in \mathbb{C}^{M p \times 1}
$$

where $\mathbf{S}_{M}=\left[\mathbf{S}^{T}[i] \cdots \mathbf{S}^{T}[i-M+1]\right]^{T}$ and $\boldsymbol{\eta}_{M}=\left[\boldsymbol{\eta}^{T}[i] \cdots \boldsymbol{\eta}^{T}[i-M+1]\right]^{T}$.

We define that $c_{j}[i]=c\left(\left(i+\frac{j}{p}\right) T\right)$. The physical channel vector is defined as $\mathbf{c}=\left[\mathbf{c}^{T}[0] \cdots \mathbf{c}^{T}[\tilde{L}]\right]^{T} \in \mathbb{C}^{(\tilde{L}+1) p \times 1}$, where $\mathbf{c}[l]=\left[c_{0}[l] \cdots c_{p-1}[l]\right]^{T}$. The total channel can be decomposed as $\mathbf{h}=\mathbf{G c}$, where the Nyquist pulse matrix $\mathbf{G} \in$ $\mathbb{R}^{L_{h} p \times(\tilde{L}+1) p}$ is a Toeplitz matrix whose first row is $[g(-\tilde{N} T) 0 \cdots 0]^{T} \in \mathbb{R}^{1 \times(\tilde{L}+1) p}$ and first column is $[g(-\tilde{N} T) \cdots g(\tilde{N} T) \quad 0 \cdots 0]^{T} \in \mathbb{R}^{L_{h} p \times 1}$. Then, we can rewrite (4) as $\mathbf{r}_{M}=\mathbf{S}_{M} \mathbf{G c}+\boldsymbol{\eta}_{M}$. Our goal is to estimate the unknown physical channel $\mathbf{c}$ from $\mathbf{r}_{M}$ rather than the total channel $\mathbf{h}$ using knowledge of the pulse shape $\mathbf{G}$ and a few training symbols $\mathbf{S}_{M}$.

\section{Semi-blind channel estimation method}

First, we consider the LS estimation of the physical channel. The LS estimation $\hat{\mathbf{c}}_{\mathrm{LS}}$ is obtained by minimizing the squared error $\left\|\mathbf{r}_{M}-\mathbf{S}_{M} \mathbf{G} \hat{\mathbf{c}}\right\|^{2}$. The estimation $\hat{\mathbf{c}}_{\mathrm{LS}}$ satisfies the following equation: 


$$
\mathbf{S}_{M} \mathbf{G} \hat{\mathbf{c}}_{\mathrm{LS}}=\mathbf{r}_{M} .
$$

If $\mathbf{S}_{M} \mathbf{G}$ has full column rank, the LS estimation is obtained by

$$
\hat{\mathbf{c}}_{\mathrm{LS}}=\left(\mathbf{G}^{H} \mathbf{S}_{M}^{H} \mathbf{S}_{M} \mathbf{G}\right)^{-1} \mathbf{S}_{M}^{H} \mathbf{G}^{H} \mathbf{r}_{M} .
$$

Note that $\mathbf{S}_{M} \mathbf{G}$ has full column rank only if $M \geq \tilde{L}+1$, and the matrix $\mathbf{S}_{M}$ consists of $L_{h}+M-1=2 \tilde{N}+\tilde{L}+M$ training symbols. Thus, the minimum number of training symbols required for $(6)$ is $N_{\mathrm{LS}}=2(\tilde{N}+\tilde{L})+1$, which increases as the channel IR length $L$ increases or the packing ratio $\gamma$ decreases.

Next, we consider a blind estimation method called subchannel matching [6]. Subchannel matching determines the subchannels $\hat{h}_{n}[k]$ that satisfy the relationship $\hat{h}_{n}[k] * r_{m}[k]=\hat{h}_{m}[k] * r_{n}[k]$ in noise-free environments, where $*$ represents convolution. This leads to the problem $\hat{\mathbf{h}}=\arg \min _{\|\mathbf{h}\|=1} \mathbf{h}^{H} \boldsymbol{\Psi} \mathbf{h}$, where $\boldsymbol{\Psi}=\mathbf{E} \boldsymbol{\Phi} \mathbf{E}^{H}$. Here, $\boldsymbol{\Phi}$ is an $L_{h} p \times L_{h} p$ block matrix whose $(i, j)$ th block is $\sum_{l \neq i} \mathbf{R}_{l l}$ for $i=j$ and $-\mathbf{R}_{j i}$ for $i \neq j$, where $\mathbf{R}_{i j}=E\left[\tilde{\mathbf{r}}_{i}[k] \tilde{\mathbf{r}}_{j}^{H}[k]\right]$ and $\tilde{\mathbf{r}}_{j}[i]=\left[r_{j}[i] r_{j}[i-1] \cdots\right.$

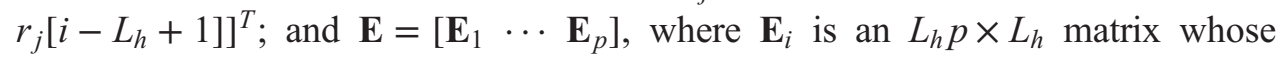
$j$ th column is $\left[\mathbf{0}_{1 \times(j-1) p} \mathbf{e}_{i} \mathbf{0}_{1 \times\left(L_{h}-j\right) p}\right]^{T}$, and $\mathbf{e}_{i}$ is a $1 \times p$ unit vector whose $i$ th entry is 1 . By using the decomposition $\mathbf{h}=\mathbf{G c}$, this problem can be modified to estimate the physical channel as [6]

$$
\hat{\mathbf{c}}_{\text {blind }}=\arg \min _{\|\mathbf{c}\|=1} \mathbf{c}^{H} \mathbf{G}^{H} \mathbf{\Psi G c} .
$$

Applying the eigenvector decomposition to $\mathbf{G}^{H} \mathbf{\Psi G}, \hat{\mathbf{c}}_{\text {blind }}$ can be found from the eigenvector corresponding to the minimum eigenvalue. We will show in our simulation that the performance of the blind method is unsatisfactory when channel IR $L$ is long.

Now, we propose a semi-blind method that combines the LS method in (5) with the blind method in (7). We expect that the proposed method can improve the performance of the blind method by using fewer training symbols than the LS method. It is known that if the subchannels share no common zeros, the minimum of $\mathbf{c}^{H} \mathbf{G}^{H} \mathbf{\Psi G c}$ in (7) is zero in noise-free environments. Thus, we rewrite the blind estimation as $\sqrt{\boldsymbol{\Lambda}} \mathbf{V}^{H} \mathbf{G c}=\mathbf{0}$, where $\boldsymbol{\Lambda}$ is a diagonal matrix whose diagonal entries are the eigenvalues of $\boldsymbol{\Psi}$, and the columns of $\mathbf{V}$ are the corresponding eigenvectors. Then, we obtain the following semi-blind estimation:

$$
\left[\begin{array}{c}
\mathbf{S}_{M} \\
\beta \sqrt{\Lambda} \mathbf{V}^{H}
\end{array}\right] \mathbf{G} \hat{\mathbf{c}}_{\text {semi }}=\left[\begin{array}{c}
\mathbf{r}_{M} \\
\mathbf{0}
\end{array}\right]
$$

where $\beta \geq 0$ is the weighting factor. Solving (8) by a numerical method such as Gaussian elimination, we obtain the semi-blind estimate $\hat{\mathbf{c}}_{\text {semi }}$. In (8), because $M \geq 1$, the minimum number of the training symbols is $N_{\text {semi }}=2 \tilde{N}+\tilde{L}+1$, which is less than $N_{\mathrm{LS}}$. Finally, we appropriately determine $\beta$ by proposing the following simple method:

$$
\beta=\frac{\left\|\mathbf{S}_{M}\right\|_{F}}{\left\|\sqrt{\Lambda} \mathbf{V}^{H}\right\|_{F}}
$$

where $\|\cdot\|_{F}$ represents the Frobenius norm of a matrix. The choice of (9) attempts to make the contribution of the blind estimation equal to that of LS. 
The computational complexity of the proposed method is dominated by the computation of the eigenvalue decomposition of $\boldsymbol{\Psi}$ and solving (8). The complexity of these computations is proportional to $(\tilde{L}+1)^{3} p^{3}$, which is almost the same as that of (6) and (7).

\section{Simulation}

We evaluate the performance of the proposed semi-blind method in (8) by computer simulation. The estimation performance is measured by the normalized mean square error (NMSE) averaged over $I$ trials

$$
\mathrm{NMSE}=\frac{\sum_{i=1}^{I}\left\|\mathbf{G} \hat{\mathbf{c}}_{i}-\mathbf{h}_{i}\right\|^{2}}{\sum_{i=1}^{I}\left\|\mathbf{h}_{i}\right\|^{2}}
$$

where the subscript $i$ indicates the $i$ th trial. Unless otherwise stated, parameters used in the simulation are as follows: modulation scheme QPSK, length of physical channel IR $L=10$, roll-off factor $\alpha=0.7, E_{b} / N_{0}=30 \mathrm{~dB}$, pulse truncation length $N=3$, and number of trials $I=10^{5}$. The correlation matrices $\mathbf{R}_{i j}$ are obtained by averaging 200 received samples.

First, we compare the proposed semi-blind method with the LS method in (6). Fig. 2(a) shows the NMSE performances versus $E_{b} / N_{0}$ for various values of the packing ratio $\gamma$ and the roll-off factor $\alpha$, where both methods use $N_{\mathrm{LS}}=2(\tilde{N}+\tilde{L})+1$ training symbols. It can be observed that the proposed semiblind method is superior to the LS method in all cases. In Fig. 2(b), the effect of the number of training symbols is shown, where the minimum required numbers of training symbols are $N_{\mathrm{LS}}=31, N_{\text {semi }}=20$ for $\gamma=0.8$ and $N_{\mathrm{LS}}=41, N_{\text {semi }}=26$ for $\gamma=0.6$. For reference, we also plotted the NMSE performances obtained by the optimum $\beta$, which is determined by an exhaustive search over a range of values $\beta \in[0,100]$. The semi-blind method can achieve a lower NMSE using a fewer number of training symbols compared to the LS method. Although the choice of $\beta$ in (9) is useful, there is little room for improvement.

Next, we compare the proposed semi-blind method, which uses the minimum number of training symbols $N_{\text {semi }}=2 \tilde{N}+\tilde{L}+1$, with the blind method in (7). In Fig. 3(a), the effect of the packing ratio $\gamma$ is illustrated. The semi-blind method is

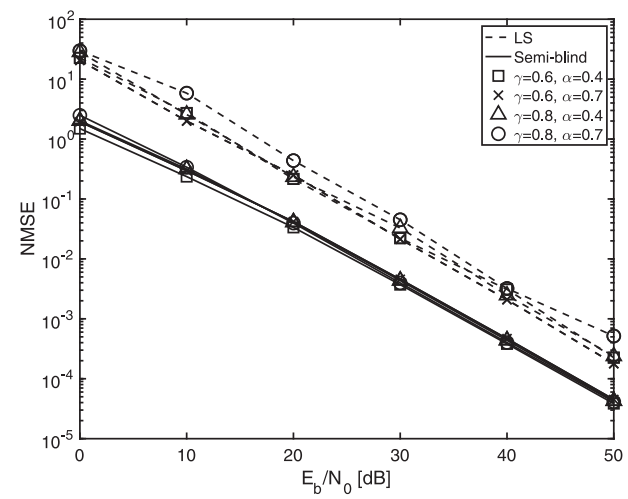

(a) Effect of FTN parameters.

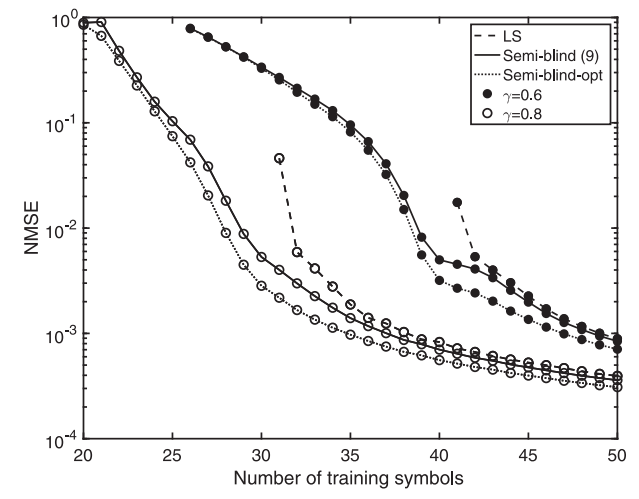

(b) Effect of the number of training symbols.

Fig. 2. Performance comparison with the LS method. 


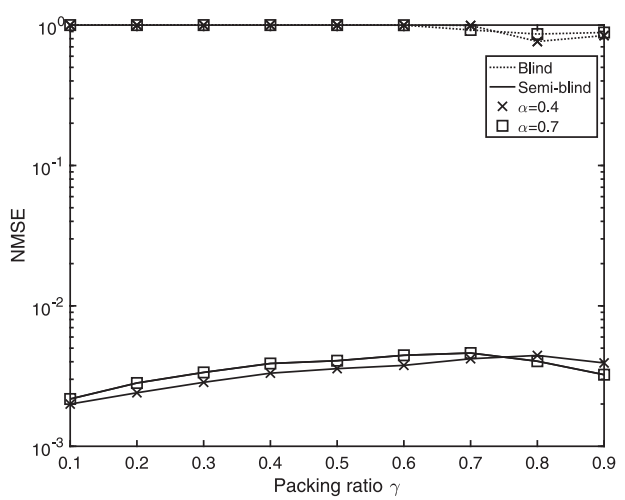

(a) Effect of FTN parameters.

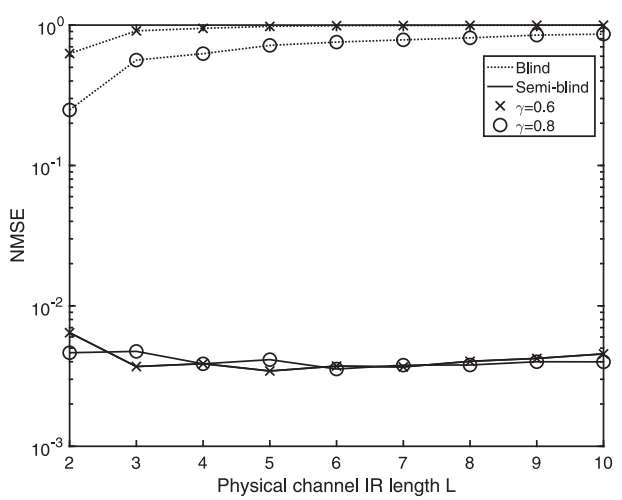

(b) Effect of channel IR length $L$.

Fig. 3. Performance comparison with the blind method.

significantly superior to the blind method. As for the semi-blind method, a lower $\gamma$ tends to exhibit a better NMSE performance. This might be because the choice of $\beta$ in (9) tends to be closer to the optimum $\beta$ as $\gamma$ decreases. Fig. 3(b) shows the effect of the IR length of the physical channel $L$. The performance of the blind method degrades significantly as $L$ increases, while that of the semi-blind method is almost independent of $L$.

\section{Conclusion}

In this paper, we proposed a semi-blind channel estimation method for FTN signaling, which is a combination of a subchannel matching based blind method and the LS method using training symbols. Our simulation results show that the proposed method requires fewer training symbols than LS, and its performance is not influenced significantly by the packing ratio and the length of the physical channel impulse response, unlike the blind method. It would be interesting to use the channel estimate obtained by the semi-blind method as an initial estimate for the iterative estimation methods in $[4,5]$ to achieve further performance improvement. 